\title{
Gestión del mantenimiento a interruptores de potencia. Estado del arte
}

\author{
Management maintenance in high voltage circuit breakers. State of the art \\ Israel Gondres Torné ${ }^{1 *} \quad$ Santiago Lajes Choy ${ }^{1} \quad$ Alfredo del Castillo Serpa ${ }^{2}$ \\ Recibido 24 de noviembre de 2016, aceptado 31 de mayo de 2017 \\ Received: November 24, $2016 \quad$ Accepted: May 31, 2017
}

\begin{abstract}
RESUMEN
En el presente trabajo se realiza una revisión bibliográfica de la gestión del mantenimiento enfocado a la confiabilidad y a las fallas en interruptores de potencia. Se valoran los posibles parámetros a utilizar en la gestión del mantenimiento de acuerdo con los estudios internacionales, a las normas y procedimientos de operación vigentes en las subestaciones eléctricas en Cuba, así como a la existencia real de equipos de medición en las mismas. Los posibles parámetros se desglosan en pruebas de funcionamiento, revisiones visuales y en la confiabilidad operacional. Finalmente se propone como herramienta fundamental la inteligencia artificial para la toma de decisiones en la gestión del mantenimiento de acuerdo a la integración de las diferentes variables.
\end{abstract}

Palabras clave: Confiabilidad, fallas, inteligencia artificial, interruptores de potencia, mantenimiento.

\begin{abstract}
The present work includes a bibliographic review of maintenance management focused on reliability and failures in high voltage circuit breakers. The possible parameters to be used in managing maintenance are evaluated according to the findings of international studies, standards and current operating procedures used by Cuban electrical substations, as well as the availability of measuring equipment in said substations. The possible parameters are broken down in functioning tests, visual reviews, and operational reliability. Finally, Artificial Intelligence is proposed as a fundamental tool for decision making in maintenance management according to the integration of the analyzed variables.
\end{abstract}

Keywords: Artificial intelligence, failure, high voltage circuit breaker, maintenance, reliability.

\section{INTRODUCCIÓN}

Actualmente importantes revistas científicas y congresos internacionales del Instituto de Ingenieros Eléctricos y Electrónicos (IEEE por sus siglas en inglés); grupos de trabajos de asociaciones profesionales como el Consejo Internacional en Grandes Sistemas Eléctricos de Alta Tensión (CIGRE por sus siglas en francés) y el Instituto Americano de Normas Nacionales (ANSI por sus siglas en inglés), así como editoriales prestigiosas como Wiley \& Sons, DEStech, Elseviers, PrenticeHall y CRC Press se han dedicado a la divulgación de los temas relacionados con la confiabilidad y

\footnotetext{
1 Departamento de Ingeniería Eléctrica. Universidad de Camagüey "Ignacio Agramonte Loynaz". Circunvalación Norte e/ Camino Viejo de Nuevitas y Ave. Ignacio Agramonte. C.P. 74650. Camagüey, Cuba.

E-mail: igondrest@gmail.com; santiago.lajes@reduc.edu.cu

2 Centro de Estudios de Matemática para las Ciencias Técnicas. Universidad Tecnológica de la Habana”. Calle 14, N 11901. Entre 119 y 129. Marianao. La Habana, Cuba. E-mail: acastillo@cemat.cujae.edu.cu

* Autor de correspondencia. E-mail: israel.gondres@reduc.edu.cu
} 
mantenimiento de los interruptores de potencia de forma general.

El interruptor es un dispositivo destinado al cierre y apertura de la continuidad de un circuito eléctrico, bajo carga y en condiciones normales, el mismo debe ser capaz de interrumpir corrientes eléctricas de diferentes capacidades, pasando desde las corrientes capacitivas de varios cientos de amperes y las inductivas de varias decenas de $\mathrm{kA}$, por ello asegurar su buen funcionamiento es de vital importancia para cualquier sistema eléctrico de potencia [1].

Existen diferentes formas de clasificar los interruptores; sin embargo, durante el desarrollo de las tecnologías, los factores principales que han influido en los parámetros de diseño son el medio aislante y el de interrupción, así como los métodos para efectuar la extinción del arco eléctrico [2].

\section{NORMAS INTERNACIONALES}

Las funciones principales de un interruptor de potencia están dadas por: cerrado debe ser un conductor ideal, abierto debe ser un aislador ideal, en posición cerrado debe ser capaz de interrumpir la corriente a la que fue diseñado, rápidamente y en cualquier instante, sin producir sobretensiones peligrosas que afecten el sistema y en posición abierto debe ser capaz de cerrar rápidamente y en cualquier instante, bajo corrientes de falla sin soldar los contactos por las altas temperaturas.

Existen otras funciones y características de los interruptores de potencia relacionados con los esfuerzos eléctricos, mecánicos, térmicos y químicos que deben soportar para mantener su confiabilidad, estas se explican en las normas internacionales que se muestran a continuación.

La norma internacional ANSI C37.06 presenta los valores nominales permisibles de operación para todos los interruptores mayores de $1 \mathrm{kV}$ (tensión nominal y tensión máxima de diseño, corriente nominal, frecuencia nominal, presión nominal de operación del gas para maniobra e interrupción, capacidad interruptiva y momentánea nominal, etc.); así como el tiempo máximo de la operación de apertura y cierre del interruptor, entre el instante de energización de la bobina de apertura hasta la separación física de los contactos principales de arco bajo ciertas condiciones específicas [3].

La norma internacional IEC 62271-100 es solamente aplicable a interruptores de tres polos para uso en sistemas trifásicos e interruptores de un polo para sistemas monofásicos, también para los dispositivos que operan los interruptores de potencia y sus equipos auxiliares, y no es aplicable para el mecanismo de cierre de los interruptores, los que dependen del manual de operación y no de esta norma. Por otro lado, se especifican las distintas pruebas de interruptores y los valores estándar de la tensión transitoria de restablecimiento [4].

Mientras que la norma internacional IEEE Std C37.010 es una guía que aborda las aplicaciones de interruptores de potencia por encima de $1 \mathrm{kV}$ para uso comercial, industrial y otras instalaciones, así como las diferentes condiciones de servicio teniendo en cuenta las posibles afectaciones de la temperatura, los esfuerzos dieléctricos y los tiempos de recierre; también detalla los cálculos de las corrientes de cortocircuitos y otros parámetros [5].

El incumplimiento de las funciones principales en los interruptores de potencia provoca graves consecuencias en los elementos del sistema o en su funcionamiento de forma general. Si el interruptor es incapaz de interrumpir la corriente de cortocircuito durante el tiempo establecido, esta puede adquirir valores extremadamente superiores a las corrientes nominales y las tensiones disminuir a valores intolerables provocando calentamientos excesivos y la temperatura de los conductores elevarse rápidamente, lo que puede deteriorar el aislamiento protector, como destruir el equipo completamente.

Tomando en cuenta que la continuidad del servicio eléctrico depende, en gran parte, de las subestaciones y que es un componente importante de los sistemas eléctricos de potencia y dentro de ellas, los interruptores de potencia, es necesario aplicarles una adecuada gestión de mantenimiento.

Se han realizado varias interpretaciones de las fallas en los equipos y elementos de las redes eléctricas, definiendo como falla a la pérdida de la función o funciones requerida de un equipo; sin embargo, lo más aceptado por la comunidad internacional es 
lo que se define por la Comisión Electrotécnica Internacional (IEC por sus siglas en inglés) en el caso de los interruptores de potencia referido a fallas mayores y menores [6].

También se definen otro tipo de fallas como: la falla eléctrica, la cual es atribuible a la aplicación de un esfuerzo eléctrico al circuito principal del interruptor y/o a los circuitos auxiliares y de control, mientras que la falla mecánica se refiere a la falla de algún elemento mecánico asociado al interruptor [7].

La clasificación por origen de la falla está hecha en fallas mecánicas y eléctricas según CIGRE, teniendo que el 70,3\% de las fallas mayores y el $85,6 \%$ de las fallas menores tienen origen mecánico. El $19,1 \%$ de las fallas mayores y el $11,7 \%$ de las fallas menores tienen origen eléctrico concerniente a los circuitos auxiliares.

La causa de las fallas es fundamentalmente el diseño (45,3\% para fallas mayores y $52,5 \%$ para fallas menores), siendo la gran mayoría de fallas de los interruptores producidas por fallas en el mecanismo de operación y en los circuitos auxiliares; sin embargo, no se pueden dejar de analizar y estudiar las otras partes que de una forma u otra inciden en el buen funcionamiento de los mismos.

Por lo que se puede afirmar que la mayor incidencia de fallas está en las partes mecánicas, y en este caso particular al no poder incidir directamente en el diseño que es la causa principal de ello, pues se hace énfasis en la gestión del mantenimiento enfocado a los contactos principales, que es donde ocurre el proceso de conmutación así como los diferentes esfuerzos eléctricos, mecánicos y térmicos.

\section{ASPECTOS TEÓRICOS DE LA TOMA DE DECISIONES PARA EL MANTENIMIENTO}

En el diccionario de la IEEE se plantea que el mantenimiento, no es más que el acto de preservar o mantener las condiciones de un equipo para su correcta operación, o sea, la combinación de todas las acciones técnicas y administrativas que incluyen acciones de supervisión, que puedan mantener o restaurar el estado para el cual cumple con las funciones afines a su desempeño un equipo o sistema determinado [8].
Las tradicionales formas del mantenimiento han cambiado en el transcurso de los años, siendo el mantenimiento preventivo y predictivo con sus diferentes acepciones los más usados en el mundo, donde los equipos son inspeccionados y revisados para fijar los intervalos correspondientes. Por ello es necesario usar otras técnicas no invasivas que ayudan a determinar su estado; ya que el costo completo de una revisión o uso de dichas técnicas está entre un tercio y un medio del precio de un interruptor de potencia nuevo [9].

Otra forma de gestionar el mantenimiento es el llamado por los especialistas Predictivo o Basado en la Condición, el mismo consiste en inspeccionar los equipos a intervalos regulares y tomar acción para prevenir las fallas o evitar las consecuencias de las mismas según condición, dichos intervalos están puestos por los fabricantes basados en experiencias anteriores y no expresan datos precisos sobre sus interruptores porque muchas veces esas informaciones son estratégicas. Dicho sistema de gestión incluye tanto las inspecciones objetivas (con instrumentos) y subjetivas (con los sentidos), como la reparación del defecto (falla potencial). Su propósito es predecir con precisión la condición del interruptor, sin tener que abrirlo para su inspección [10].

Mientras que el Mantenimiento Centrado en la Confiabilidad permite de forma eficiente, optimizar los procesos de producción y disminuir al máximo los posibles riesgos sobre la seguridad personal y el ambiente, que traen consigo los fallos de los activos en un contexto operacional específico.

Por ello la gestión del mantenimiento en interruptores de potencia puede tomar varias maneras de complementarse entre sí, o sea, mediante pruebas técnicas y revisiones visuales para verificar la conformidad de cada una de las propiedades eléctricas y mecánicas; por medio de análisis estadísticos para descubrir los componentes con falla o ayudar a crear un modelo probabilístico del envejecimiento en los interruptores para su mantenimiento, también mediante análisis de confiabilidad operacional para valorar ciertos aspectos de funcionamiento in situ; por último y no menos importante el monitoreo de la condición para la vigilancia continua del interruptor por medio de múltiples transductores controlados 
por una computadora o mediante elementos que brindan las mediciones de diferentes parámetros. Todo ello se puede integrar mediante la inteligencia artificial y brindar mejores resultados desde todos los puntos de vista.

\section{Pruebas que inciden en la gestión del mantenimiento centrado en la confiabilidad}

El estudio de los problemas asociados a los contactos eléctricos es algo común en varios congresos nacionales e internacionales, uno de los más notables es la Conferencia Holm de Contactos Eléctricos de la IEEE que tiene lugar anualmente en Estados Unidos de América o Canadá, así como la Conferencia Internacional de Contactos Eléctricos (ICEC por su siglas en inglés) que tiene sede en varios países y se realiza cada dos años.

Entre las pruebas que inciden en la confiabilidad de los interruptores está la de resistencia estática de contactos, la que proporciona un valor de referencia para todos los tipos de conexiones de alta intensidad. Mientras que la prueba de resistencia dinámica de contacto inicialmente estaba enfocada a medir la resistencia durante una operación lenta de apertura del interruptor. Por lo que se realizan a baja velocidad, para que no esté presente la separación parcial del contacto, haciendo que sea más fácil medir la resistencia con una corriente continua de $100 \mathrm{~A}$ [11].

Otra prueba está relacionada con la resistencia de aislamiento, de acuerdo con los procedimientos de las pruebas que se realizan actualmente por la Empresa de Construcciones de la Industria Eléctrica (ECIE) se dan varias consideraciones a tener en cuenta en relación a la nueva generación de interruptores. Entre ellas se hace relación a los catálogos de los fabricantes de los interruptores GL312 y GL314 que en las recomendaciones no aparece la prueba de resistencia de aislamiento como una prueba rutinaria para diagnosticar el estado del interruptor; sin embargo, no se descarta esta prueba ya que ofrece resultados y elementos al personal encargado de decidir el estado de un equipo en un momento determinado. La comprobación de la resistencia del aislamiento se realiza aplicando una tensión de $5 \mathrm{kV}$ entre la parte superior del aislador de apoyo y el otro extremo (masa, tierra) el valor mínimo de resistencia será de $\geq 1.000 \mathrm{M} \Omega$ a $20^{\circ} \mathrm{C}$, como condición el interruptor estará en posición abierta, por lo que se medirán dos elementos en paralelo que serán el aislador de apoyo propiamente y el vástago del contacto móvil [12].

Por otro lado, las pruebas de tiempos de operación tienen como propósito analizar el desempeño del mecanismo de operación de los interruptores, en función de los tiempos de operación, en sus diferentes formas de maniobra, así como la verificación del sincronismo de sus tres fases y de los contactos de una misma fase $[2,13]$.

Varios autores muestran otras pruebas que se realizan indistintamente según los tipos de interruptores y de acuerdo con la existencia física de instrumentos de última tecnología, ello se puede consultar más detalladamente en las siguientes referencias [9, 14-15].

\section{Revisiones e inspecciones visuales a interruptores de potencia}

Una de las recomendaciones dada por los fabricantes de interruptores de potencia es la realización periódica de inspecciones visuales debido al importante rol que juegan los mismos en los sistemas de transmisión y de distribución. Debido a que el interruptor es una caja negra, la única manera de evaluar su condición con certeza es desmontarlo; sin embargo, ello puede ser muy costoso, especialmente si no es necesario. Por ello se tratan de aplicar una serie de pruebas, inspecciones y revisiones para recolectar suficiente información sobre la verdadera condición del interruptor, la que a su vez permite tomar una decisión para proceder con el mantenimiento del mismo a tiempo. Estas acciones pueden abarcar desde una simple lectura, como un contador de operaciones, hasta una inspección completa, la cual incluye todos los gabinetes, la calefacción, los terminales primarios, el aterramiento, etc.

De acuerdo con las normas estipuladas por la ECIE, las inspecciones se deben realizar no menos de dos veces en el año, o sea, una inspección profunda visual. En ella se debe revisar el estado general del interruptor, precisando el estado de las conexiones y de la porcelana que forman las columnas polares, además si se nota un grado alto de contaminación, o cualquier indicio de falsos contactos que provoquen puntos calientes; el estado y posición que indiquen los densímetros en cada 
polo (zona de colores), verificar el funcionamiento de la calefacción permanente en el gabinete de control y la lectura del cuenta operaciones para registrar el acumulado de las operaciones.

Estas inspecciones tienen un alto grado de subjetividad e incertidumbre ya que es el operador el que de forma visual valora el estado del equipamiento y en la planilla correspondiente solo refiere si está bien o mal, por lo que no existen términos medios o un rango de evaluación de forma tal que pueda darle un seguimiento más acertado a su estado real, mientras que las lecturas del contador y las corrientes que circulan no son analizadas como corresponde de acuerdo con la curva característica de desgaste mecánico y la expresión matemática que rige el desgaste eléctrico, las que están tanto en la literatura internacional como en los catálogos de fabricantes.

Por lo que se observa que las principales pruebas e inspecciones visuales, así como las tareas de mantenimiento y reparaciones no están dirigidas principalmente a las piezas que están sometidas al desgaste y envejecimiento; donde los factores de influencia decisivos están dados por el número de conmutaciones en cortocircuito, la frecuencia de conmutación y el tiempo de servicio. Teniendo en cuenta estas consideraciones y dando respuesta a las exigencias de la tecnología existente, así como a la existencia de equipos se determina analizar en su conjunto las pruebas relacionadas con la medición del aislamiento, la resistencia de contacto y la resistencia de las bobinas.

\section{ESTUDIOS REALIZADOS A INTERRUPTORES DE POTENCIA}

Los estudios de confiabilidad y análisis de fallas siempre van asociados a análisis estadísticos con el fin de tomar varias directrices tanto los fabricantes como los usuarios, los primeros para mejorar el diseño, y los otros para incidir en el sistema de gestión de mantenimiento a implementar.

El primer estudio se realizó a 77.892 interruptores de todos los tipos en 102 empresas de 22 países por el grupo de trabajo 13.06 de CIGRE en el período de 1974 a 1977 [16], en el segundo estudio se analizaron 70.708 interruptores de Hexafloruro de Azufre (SF6) [17] en el que se hizo un análisis profundo de la naturaleza de las fallas de mayor y menor importancia de acuerdo con la clasificación dada por [6], obteniendo como resultado que las viejas tecnologías tienen más posibilidades de fallas mayores que las de SF6 y estas, a su vez, más posibilidades de fallas menores que las tecnologías antiguas, solamente el $6,1 \%$ de la fallas mayores y el $13,7 \%$ de la fallas menores se encontraron durante el mantenimiento, lo que indica que no fue efectivo para encontrar los defectos y posibles fallas. Por otra parte, el 2,8\% de las fallas mayores y el $2,6 \%$ de la fallas menores fue atribuida a un mantenimiento incorrecto, además de reafirmar que el mantenimiento no ha sido efectivo en evitar y disminuir dichas fallas.

Recientemente se culminó el tercer estudio por el Comité A3.06 de CIGRE en el período de 2004 a 2007 [18] en el que se analizaron 281.090 interruptores de SF6 de 83 compañías en 26 países, realizando diferentes análisis estadísticos relacionados con la frecuencia en función del nivel de tensión, año de instalación, tecnología, lugar, mantenimiento y análisis de falla según los modos, origen, causas, subcomponentes fallados y mediciones, dando como resultado que la principal causa de falla es el desgaste, por lo que recomiendan una revisión crítica de las actuales estrategias y frecuencias de mantenimiento utilizadas por las diferentes compañías eléctricas en aras de evitar y prevenir fallas catastróficas.

A pesar de la amplitud y profundidad de dichos estudios que ayudan tanto a los fabricantes a optimizar el diseño como a los usuarios a comprender el comportamiento de los interruptores de potencia en las redes eléctricas, así como a la operación, mantenimiento y monitoreo efectivo de los distintos parámetros o elementos para un buen desempeño del sistema, no indican la forma, vías o procedimientos para realizar una gestión adecuada de dichos mantenimientos.

Por otro lado, también se han realizado otras investigaciones en diferentes países; en México se analizaron y estudiaron las fallas de una población de alrededor de 3.556 interruptores durante el período de 21 años iniciando en 1981 y concluyendo en el 2001 [2]; se realizó un análisis estadístico para encontrar puntos de mejora, tanto en la operación como en el mantenimiento de los interruptores 
instalados, establecer las estrategias y las acciones a seguir para incrementar la confiabilidad y disponibilidad de los mismos en sus instalaciones. El estudio demostró que uno de los puntos de mayor relevancia, en cuanto a fallas, es la falta de mantenimiento y la programación inadecuada de intervención en los equipos, así como a la falta de disponibilidad y repuestos de los mismos.

En Alemania durante un estudio de las redes eléctricas se incluyó también el análisis de la fatiga de alrededor de 1.800 interruptores de potencia de acuerdo con las corrientes de carga y cortocircuito, o sea, los análisis se enfocaron al desgaste eléctrico del mismo a partir de las corrientes de fallas [19]; sin embargo, en dicho estudio no se realiza ninguna alusión a la gestión del mantenimiento para evitar dicho desgaste.

En Suecia y Finlandia se analizaron las fallas de alrededor de 1.546 interruptores [20], concluyendo que la frecuencia de fallas aumentó con el nivel de voltaje y que la cantidad de operaciones influía directamente con la confiabilidad de dichos interruptores. En este estudio se utilizaron los datos para modelar estadísticamente la confiabilidad pero no se tuvo en cuenta la utilización de estrategias para dirigir el mantenimiento.

También en China el Instituto de Investigaciones de Potencia llevó a cabo un estudio entre 1999 y 2003 de varios interruptores de potencia mayor de $63 \mathrm{kV}$ [21], al igual que en Japón se analizó la confiabilidad y los esfuerzos de un numeroso grupo de interruptores [22]. Ambos estudios no realizaron un análisis sobre la gestión del mantenimiento.

Otros estudios han demostrado los beneficios directos del diagnóstico y monitoreo de la condición en equipos y sistemas referidos a la reducción de los costos de mantenimiento, incremento de la confiabilidad y disponibilidad de los equipos así como el aumento de la vida útil de los mismos [23-24].

En el caso específico de los interruptores se ha llegado a un consenso general por los grupos de trabajo 13.06 y 23.10 de CIGRE en la importancia del monitoreo de los siguientes elementos: posición del contacto (desplazamiento y velocidad), continuidad de los circuitos de disparos, aislamiento y medio de extinción del arco, desgaste de los contactos basados en el acumulado de interrupciones, tiempos de operación, tiempo de carga del mecanismo, así como otros parámetros [25].

Algunos estudios han sido dirigidos al análisis de vibraciones durante la operación, ya que permite la detección de anomalías mecánicas en el mecanismo o en la cámara de interrupción de los interruptores SF6 de alta tensión, y puede registrar cualquier señal analógica relevante al análisis de la condición del interruptor, tal como el desplazamiento y la posición del contacto [26, 27, 28, 29].

Sin embargo, en las subestaciones eléctricas en Cuba se utiliza el término mantenimiento de forma general sin tener en cuenta las filosofías mundiales que rigen la actividad, se hace un acercamiento al tipo de mantenimiento planificado preventivo teniendo solamente en cuenta los criterios de los fabricantes, que en algunos afirman que son libres de mantenimiento o en el mejor de los casos recomiendan que se realicen mantenimientos cada seis años o más sin tener en cuenta las características propias de cada territorio; en el caso particular de los interruptores tanto para $110 \mathrm{kV}$ como para $220 \mathrm{kV}$ lo ejecutan solamente de acuerdo con las normas nacionales [30-31].

En dichas normas se chequean varios parámetros pero de forma aislada y no se tienen en cuenta como un sistema, por lo que se dificulta tomar una decisión con tantos datos, la complejidad mayor la constituye la incertidumbre y ambigüedad en la evaluación de las revisiones de algunas variables, el análisis estadístico de las pruebas, así como los procesos de desgaste que ocurren debido a las distintas operaciones a corrientes nominales o de cortocircuitos. Por ello es extremadamente arriesgado tomar la decisión utilizando únicamente la experiencia práctica o un procedimiento de acuerdo con las indicaciones del fabricante, sobre todo cuando existan parámetros con diferentes evaluaciones de regular, irregularidades en la toma o falta de algunos datos históricos de las pruebas por situaciones ajenas a la voluntad del especialista, así como a las condiciones de operación del sistema eléctrico.

Por tanto, utilizar la inteligencia artificial para obtener un modelo que permita perfeccionar la gestión del mantenimiento parcial para decidir cuándo efectuar el mantenimiento o elaborar los 
planes de reparaciones en los interruptores de potencia es de gran importancia y necesidad para las empresas eléctricas.

\section{APLICACIÓN DE TÉCNICAS DE INTELIGENCIA ARTIFICIAL}

Los Sistemas Eléctricos de Potencia (SEP) no han permanecido ajenos a la implementación y uso de la inteligencia artificial en sus distintos elementos, o sea, ha abierto un nuevo camino hacia el desarrollo de la protección de sistemas y máquinas eléctricas, imponiéndose, debido a su versatilidad, alta precisión y nivel de exactitud, con gran auge sobre los métodos tradicionales de protección; las técnicas más usadas hasta el momento son las relacionadas con los llamados sistemas híbridos, donde intervienen las redes neuronales, lógica difusa, algoritmos genéticos y conjuntos aproximados.

En el caso de las redes neuronales han alcanzado gran desarrollo y alto nivel de aplicación, varias han sido dirigidas a los análisis de seguridad, predicción de la demanda, detección y diagnóstico de fallas, operación de los SEP, modelación y control de máquinas, entre otros [32-33-34].

Mientras que el uso de los algoritmos genéticos se ha ido expandiendo en los SEP [35-36] estos están inspirados en el principio darwiniano de la evolución de las especies y en la genética, o sea, son algoritmos probabilísticos que ofrecen un mecanismo de búsqueda paralela y adaptativa basada en el principio de supervivencia de los más aptos y en la reproducción.

Varios trabajos se han realizado con la lógica difusa en los SEP, tanto en las redes de distribución y transmisión [37-38], transformadores [39], generadores [40].

En el caso particular de los interruptores de potencia se han realizado varios estudios encaminados a la selección del tiempo de mantenimiento óptimo mediante el uso de la lógica difusa, se han usado diferentes vías o métodos, uno de ellos es mediante las relaciones de preferencia difusas [41].

También se han realizado estudios de los parámetros a monitorear de acuerdo a las fallas más frecuentes, utilizando matrices de fallas, de síntomas de fallas y relacional difusa, por medio de las cuales obtienen el operador difuso y las reglas de diagnóstico correspondientes, así como proponen métodos adaptativos de corrección de la matriz relacional difusa [42].

Por otro lado, algunos autores han combinado las redes neuronales y la lógica difusa para el diagnóstico de fallas en interruptores de potencia, obteniendo un modelo para la identificación de fallas a partir del conocimiento incierto y datos ambiguos, así como el mejoramiento de su precisión [43].

En otro orden también se han utilizado diferentes variables de las inspecciones visuales a los interruptores, determinando los índices de deterioro mediante la fusificación y defusificación de dichos parámetros para tomar la decisión óptima del tiempo de mantenimiento [44].

En cuanto a la filosofía de los conjuntos aproximados, se basan en la suposición que alguna información está asociada con cada objeto del universo de discurso [45-46]. El análisis de datos de los conjuntos aproximados provee un método para ganar compenetración en las propiedades de datos y se basa en los datos originales, no necesita cualquier información externa, así como no es necesaria ninguna suposición acerca de los datos, por lo que sirve para analizar tanto rasgos cualitativos como cuantitativos siendo utilizado indistintamente en los SEP [47-48].

La mayoría de las veces las técnicas se implementan de forma mixta, de manera que se aprovechen las características de cada una de ellas de forma sinérgica. La combinación de todas también contribuye a aumentar las prestaciones del sistema de diagnóstico de fallos [49-50].

Sin embargo, todavía existen potencialidades que no se explotan al máximo, tanto en la cantidad de variables o parámetros a tener en cuenta en la gestión del mantenimiento, así como en las técnicas de inteligencia artificial que han avanzado considerablemente en los últimos años, y en específico las que se combinan entre sí, por ello se podrían usar todos los beneficios inherentes a dichos sistemas híbridos, principalmente los conjuntos aproximados difusos [51-52], los que 
permitirán obtener una metodología apropiada en la gestión del mantenimiento de dichos elementos; ya que se resolvería el problema de incertidumbre de los datos obtenidos en las revisiones visuales, los cuales tienden a ser difusos mientras que, por otra parte, se concatenaría la solución de las pruebas de funcionamiento y los métodos utilizados para la determinación de la confiabilidad operacional que están íntimamente interrelacionados entre sí.

\section{CONCLUSIONES}

Se determinaron los posibles parámetros a tener en cuenta para el modelo de gestión del mantenimiento de acuerdo con los estudios realizados por CIGRE, así como a las normas y procedimientos nacionales e internacionales.

El uso de la inteligencia artificial en la gestión y solución de diversos problemas donde intervienen diferentes variables que puedan ser ambiguas o difusas, permite incluir las variables necesarias para la gestión contextualizada del mantenimiento parcial a interruptores de potencia.

Existe un amplio campo de investigación en el desarrollo de estrategias de mantenimiento que contribuyan al incremento de la confiabilidad operacional de los interruptores de potencia a partir de las filosofías mundiales del mantenimiento y a los requerimientos técnicos y operacionales de las nuevas tecnologías.

\section{REFERENCIAS}

[1] IEEE Std C37.10. IEEE Guide for Investigation, Analysis, and Reporting of Power Circuit Breaker Failures. United States of America: IEEE, 2011.

[2] A. E. Ayub, A. Hernández, E. Rosales. Manual de Interruptores de Potencia. México: CFE/CTT, 2003.

[3] ANSI C37.06. AC High-Voltage Circuit Breakers Rated on a Symmetrical Current Basis-Preferred Ratings and Related Required Capabilities. USA: American National Standards Institute, Inc. 2000.

[4] IEC 62271-100. Standard High Voltage Switchgear and Controlgear. Part 100: High-voltage alternating-current circuitbreakers. Switzerland. IEC, 2001.
[5] IEEE Std C37.010. IEEE Application Guide for AC High-Voltage Circuit Breakers Rated on a Symmetrical Current Basis. New York: IEEE, 2005. pp. 81. ISBN: 0-7381-1837-3 SH94796.

[6] IEC 60694. Common specifications for highvoltage switchgear and controlgear standards. Geneva: International Electrotechnical Commission, 2002.

[7] P. O'Connor, A. Kleyner. Practical Reliability Engineering. Fifth Edition. New Delhi: Wiley \& Sons, Ltd, 2012, pp. 512. ISBN: 978-0-470-97982-2.

[8] IEEE 100. The Authoritative Dictionary of IEEE Standards Terms. New York: IEEE, 2000, pp. 1362. ISBN: 0-7381-2601-2.

[9] Y. Fan, Q. Chenxi, Z. Xiaohui, D. Jun, P. Yujie, H. Xiaoguang. Integrated state test system of high voltage circuit breakers based on embedded technology. 6th IEEE Conference on Industrial Electronics and Applications (ICIEA). Beijing, China. 2011.

[10] M. Al-Suhaily, S. Meijer, J. Smit, P. Sibbald, J. Kanters. Criticality assessment of GIS components. International Conference on High Voltage Engineering and Application (ICHVE). New York, USA. 2010.

[11] E. Nasrallah, F. Brikci, S. Perron. Contactos de Apertura/Cierre en Interruptores de Potencia. Revista Electric Energy T\&D. 2007.

[12] J.L. Silva. C-PU 0031 Procedimiento para ejecución de pruebas de alto voltaje con $\mathrm{CD}$ a interruptores de soplo de aire tipo $\mathrm{BBb}$ de 110 y $220 \mathrm{kV}$ y de SF6. La Habana: ECIE, 2010.

[13] C. E. López. Prueba a interruptores de potencia. Tesis de diploma presentada en opción al título de Ingeniero Electricista. Universidad de San Carlos de Guatemala, Guatemala. 2008.

[14] N. Charbkeaw, T. Suwanasri, T. Bunyagul. Mechanical defect detection of SF6 high voltage circuit breaker using wavelet based vibration signal analysis. 5th International Conference on Electrical Engineering Electronics, Computer, Telecommunications and Information Technology. Thailand. 2008.

[15] H. Xiaoguang, L. Chao. Research on the condition parameter tester of high voltage circuit breakers. 3rd IEEE Conference on 
Industrial Electronics and Applications, ICIEA. Singapore. 2008.

[16] G. Mazza, R. Michaca. The first international enquiry on high voltage circuit breaker failures and defects in services. Electra No 79, pp. 21-29. 1981. ISSN: 1286-1146.

[17] CIGRÉ Working Group 13.06, "Final report of the second international enquiry on high voltage circuit-breaker failures and defects in service," Int. Council Large Elect. Syst. (CIGRÉ), Paris, France, Tech. Brochure 83, 1994.

[18] CIGRÉ Working Group A3.06, "Final report of the 2004-2007 international enquiry on reliability of high voltage equipment, Parts 1-6", Int. Council Large Electric Systems (CIGRÉ), Paris, France, Tech. Brochures 509-514, 2012.

[19] C. Neumann, G. Balzer, J. Becker, R. Meister, V. Rees, C.E. Sölver. Stress of HV circuit-breakers during operation in the networks-German utilities experience. Int. Council Large Electric Systems (CIGRÉ), Paris, France, SC13, report 13-304. 2002.

[20] T.M. Lindquist, L. Bertling, R. Eriksson. Circuit breaker failure data and reliability modelling. IET Generation, Transmission \& Distribution, pp. 813-820. 2008. ISSN: 1751-8695. URL: http://ieeexplore.ieee. org/document/4663092/ Fecha de Consulta: 15 de octubre de 2016. DOI: 10.1049/ iet-gtd:20080127

[21] S. Gao, C. Jinchun, Y. Dalu. Operating Analysis of High Voltage Circuit Breaker in 1999-2003. Electrical Equipment, Vol. 6 $\mathrm{N}^{\circ}$ 2, pp. 6-13. 2005. ISSN: 1672-2000. URL: http://en.cnki.com.cn/Article_en/ CJFDTOTAL-DLSB200502001.htm. Fecha de Consulta: 15 de noviembre de 2016.

[22] Y. Nakada, I. Takagi, M. Shin, J. Kida, M. Toyoda and H. Ito. Reliability and electrical stress survey on high voltage circuit breakers in Japan, Vol. A3, No 205, (CIGRÉ), Session 41, Paris, France, 2006.

[23] X. Jin, Z. Liujun, H. Xiaoguang, L. Liang, Z. Lei. Development of On-line Monitoring System for SF6 Circuit Breaker. 6th IEEE Conference on Industrial Electronics and Applications. New York, USA. 2011.

[24] I. Desai, J. Jamnani, S. Takalkar. Reliability improvement of Extra High Voltage substation.
Nirma University International Conference on Engineering (NUiCONE). New York, USA. 2011.

[25] M. Stanek. Model-Aided diagnosis for high voltage circuit breakers. A dissertation submitted for the degree of Doctor of Technical Sciences. Diss. ETH No 13507. Swiss Federal Institute of Technology Zurich. Zurich, Switzerland. 2000.

[26] Q. Sun, T. Cao, Y. Hou, T. Zhao. Detection and Analysis Based on the Abnormal Mechanical Vibration Signal of GIS. 2015 Fifth International Conference on Instrumentation and Measurement, Computer, Communication and Control (IMCCC). Qinhuangdao, China. 2015.

[27] H. Chunguang, L. Yulong, C. Yundong, L. Jing, C. Yuchen. Analysis on vibration and acoustic joint mechanical fault diagnosis of high voltage vacuum circuit based on wavelet packet energy relative entropy. 27th International Symposium on Discharges and Electrical Insulation in Vacuum (ISDEIV). Suzhou, China. 2016.

[28] T. Jin, N. Weihua, C. Sun. Fault diagnosis method of high voltage circuit breakers based on wavelet packet decomposition and ELM. 2016 China International Conference on Electricity Distribution (CICED). Xi'an, China. 2016.

[29] R. Quintana. "Análisis dinámico del mecanismo del interruptor de potencia GL314 de SF6 bajo régimen normal de funcionamiento". Tesis de diploma presentada en opción al título de Ingeniero Mecánico. Universidad de Camagüey "Ignacio Agramonte Loynaz". Camagüey. Cuba. 2016.

[30] J.L. Silva. C-IT HY 44. Instrucción de operación y explotación de los interruptores S1-145 F1/ 3131. La Habana: ECIE, 2012.

[31] J.L. Silva. C-IT HY 46. Instrucción de operación y explotación de los interruptores GL314. La Habana: ECIE, 2012.

[32] A. Ziani, H. Moulai. Application of artificial neural networks for electric arc extinction modeling in high voltage circuit breakers. 15th IEEE Mediterranean Electrotechnical Conference MELECON. Valletta, Malta. 2010.

[33] Q. Ben-Sheng. Research on fault diagnosis of high-voltage circuit breaker based on 
the improved BP neural network. 2nd International Conference on Information Science and Engineering (ICISE), Nanjing, China. 2010.

[34] S. Laijun, L. Mingliang, Z. Jianju, Y. Guangzhong. A new fault diagnosis method for HV circuit breakers based on wavelet packet-neural network. 6th IEEE Conference on Industrial Electronics and Applications (ICIEA). Beijing, China. 2011.

[35] T. Krontiris, G. Balzer. Assessing the effect of maintenance on high-voltage circuit breaker reliability. 11th International Conference on Methods Applied to Power Systems (PMAPS). Singapore, Singapore. 2010.

[36] O. Quintana. Aplicaciones de los algoritmos genéticos a la Ingeniería Eléctrica. Tesis presentada en opción al título de Ingeniero Electricista. Universidad de Camagüey "Ignacio Agramonte Loynaz". Camagüey, Cuba. 2012.

[37] E. Sierra. Modelo de gestión de mantenimiento por diagnóstico en redes eléctricas de distribución primaria. Tesis presentada en opción al grado científico de Doctor en Ciencias Técnicas. Universidad de Camagüey "Ignacio Agramonte Loynaz". Camagüey, Cuba. 2010.

[38] Y. Liu, C. Sing. Application of fuzzy inference systems for evaluation of failure rates of power system components. 16th International Conference on Intelligent System Application to Power Systems (ISAP). Texas, USA. 2011.

[39] H. Yann-Chang, S. Huo-Ching. Dissolved Gas Analysis of Mineral Oil for Power Transformer Fault Diagnosis Using Fuzzy Logic. IEEE Transactions on Dielectrics and Electrical Insulation, Vol. 20, No 3, pp. 974981. 2013. ISSN: 1070-9878. URL: http:// ieeexplore.ieee.org/stamp/stamp.jsp?tp $=\&$ arnumber $=6518967 \&$ isnumber $=6518934$. Fecha de Consulta: 10 de agosto de 2014. DOI: 10.1109/TDEI.2013.6518967

[40] R. Ramya, K. Selvi. A simple fuzzy excitation control system for synchronous generator. International Conference on Emerging Trends in Electrical and Computer Technology (ICETECT). Tamil Nadu, India. 2011.

[41] I. Gondres, M. Alexandrovna. Selección multicriterial de la gestión de mantenimiento en Interruptores de Potencia a través de
Relaciones de Preferencia Difusas. Seminario Internacional "La Ingeniería Eléctrica y Mecánica por un Desarrollo Sostenible". Camagüey. Cuba. 2007.

[42] H. Lingjie, W. Wei, W. Zhensheng, X. Lijie. Research on the model of HV SF6 circuit breaker fault diagnosis based on fuzzy theory. International Conference on Condition Monitoring and Diagnosis. Beijing, China. 2008.

[43] M. Hongxia, W. Honghuag. Research on fault diagnosis method of high-voltage circuit breaker based on fuzzy neural network data fusion. International Conference on Computer Application and System Modeling (ICCASM). Taiyuan, China. 2010.

[44] I. Gondres, S. Lajes, N. Rodríguez, A. Castillo. El aprendizaje bajo incertidumbre aplicado al mantenimiento de interruptores de potencia. Revista Ingeniería Energética. Volumen XXXV, Número 2, pp. 149-158. Cuba: 2014. ISSN: 1815-5901. URL: http:// rie.cujae.edu.cu/index.php/RIE/article/ view/399/401. Fecha de Consulta: 12 de abril de 2015.

[45] R. Bello, J.L. Verdegay. Los conjuntos aproximados en el contexto de la Soft Computing 1-2, Enero-Junio de 2010, RCCI, Vol. 4, pp. 5-24. ISSN 2227-1899. Disponible en: <http://rcci.uci.cu/?journ al=rcci\&page $=$ article $\&$ op $=$ view $\&$ path $\%$ $5 \mathrm{~B} \% 5 \mathrm{D}=103>$. Fecha de acceso: 14 nov. 2016.

[46] R. Bello, M. García, J.N. Pérez. Teoría de los conjuntos aproximados: conceptos y métodos computacionales. Universidad Distrital Francisco José de Caldas. Bogotá, Colombia. 2012. ISBN: 9789588723778.

[47] X. Yun-Fang, Z. Yu-Hong, C. Jin-Jin. The research on fault diagnosis of distribution network based on rough set theory. International Colloquium on Computing, Communication, Control, and Management CCCM. Sanya, China. 2009.

[48] S. Minfeng, Q. Congming. Research of a new transformer fault diagnosis expert system. China International Conference on Electricity Distribution. Shanghai, China. 2012.

[49] Z. Liying, W. Dazhi, Z. Cuiling, L. Xiaoqin. A New Fault Diagnosis Model of Electric 
Power Grid Based on Rough Set and Neural Network. Fourth International Conference on Multimedia Information Networking and Security (MINES). Nanjing, China. 2012.

[50] L. Zhi-Bin, X. Zhi-Hui. Transformer Fault Diagnosis Based on rough sets and support vector machine. Asia-Pacific Power and Energy Engineering Conference (APPEEC). Shanghai, China. 2012.
[51] H. Chih-Ching. Robust function approximation based on Fuzzy sets and Rough sets. IEEE International Conference on Fuzzy Systems, FUZZ-IEEE 2009, pp. 1250-1254. Jeju Island, Corea del Sur. 2009.

[52] Y. Qu, C. Shang, Q. Shen, N. M. Parthaláin, W. Wu. Kernel-based fuzzy-rough nearest neighbour classification. IEEE International Conference on Fuzzy Systems (FUZZ). Taipei, China Taipei. 2011. 